\title{
Producción de Hidrógeno a partir de energía solar en el campus de la UNI
}

\author{
Johnny Nahui Ortiz, Caroline Camarena Gamarra, Alejandro Mendoza Rojas \\ Universidad Nacional de Ingeniería, Av. Túpac Amaru 2010 - Rímac, Lima, Perú \\ Recibido 16 de junio del 2019, Revisado el 21 de julio de 2019 \\ Aceptado el 22 de julio de 2019
}

DOI: https://doi.org/10.33017/RevECIPeru2019.0007/

\section{Resumen}

El presente proyecto se desarrolló en la Universidad Nacional de Ingeniería (UNI), en la cual existe interés en investigar acerca de la energía del hidrógeno como posible combustible del futuro, en el contexto del aprovechamiento de las energías renovables que es uno de los pilares de la Ingeniería Ambiental constituyendo un aspecto clave en el proceso formativo de los estudiantes. El objetivo general es evaluar experimentalmente la eficiencia energética de la producción de hidrógeno a partir de energía solar fotovoltaica. Se hicieron mediciones de irradiación solar, temperatura y humedad relativa en las instalaciones de la Facultad de Ingeniería Ambiental (FIA-UNI). Se registraron valores máximos de temperatura cercanos a los $30^{\circ} \mathrm{C}$ en un día del mes de noviembre, valores mínimos de humedad relativa de $50 \%$ en un día del mes de noviembre y valores máximos de irradiación solar cercanos a los $900 \mathrm{~W} / \mathrm{m}^{2}$, en un día del mes de marzo. Se implementó un sistema de producción de electricidad a partir del recurso solar, constituido por dos módulos fotovoltaicos, dos baterías, un controlador y un inversor. Este sistema se ha implementado como módulo demostrativo para ser usado como parte del proceso formativo de los estudiantes de la FIA-UNI. Se hicieron pruebas de producción de hidrógeno mediante electrólisis, utilizando un sistema integrado equipado con una celda de combustible tipo PEM. En promedio, se obtuvo un ratio de $50.13 \mathrm{~kW} . \mathrm{h} / \mathrm{kg}$ de producción de hidrógeno a partir de energía solar fotovoltaica utilizando una celda de combustible tipo PEM en el campus de la UNI. Se anticipa un próximo trabajo de investigación utilizando un sistema híbrido integrado al sistema solar fotovoltaico.

Descriptores: Recurso Solar, Sistema Fotovoltaico, Energía Eléctrica / Electrólisis / Producción de Hidrógeno.

\begin{abstract}
The present study was carried out at the National University of Engineering (UNI) that is very interested in research work about hydrogen energy as a potential fuel for the future, within the framework of utilization of renewable energies which is a core competency for Environmental Engineering in the academic work of students. The main objective is to evaluate energy efficiency associated with hydrogen production based on solar photovoltaic energy. Measurements for solar irradiation, temperature, and relative humidity were conducted in the facilities of the Environmental Engineering Department (FIA-UNI). Maximum values for temperatures around $30^{\circ} \mathrm{C}$ were registered in November, minimum values for relative humidity around $50 \%$ were registered in November, and maximum values for solar irradiation around $900 \mathrm{~W} / \mathrm{m}^{2}$ were registered in March. An electricity production system based on solar resource was implemented and has two photovoltaic modules, two storage batteries, a controller and an inverter. The system has been implemented as a demonstrative module in order to be used as part of the academic work of the students at FIA-UNI. Test of hydrogen production through electrolysis were carried out using a systems integrated with a PEM-type fuel cell. An average ratio of $50.13 \mathrm{~kW} . \mathrm{h} / \mathrm{kg}$ of hydrogen production based on solar energy was obtained using a PEM-type fuel cell on campus at UNI. Further research is expected to be conducted using a hybrid system with integration to the solar energy system.
\end{abstract}


Keywords: Solar Resource, Photovoltaic System, Electric Power, Electrolysis, Hydrogen Production.

\section{Introducción}

En el campus de la Universidad Nacional de Ingeniería (UNI) existe interés en investigar acerca de la energía del hidrógeno como posible combustible del futuro, el contexto del aprovechamiento de las energías renovables que es uno de los pilares de la Ingeniería Ambiental constituyendo un aspecto clave en el proceso formativo de los estudiantes.

Las tecnologías desplegadas en el mundo para la producción de hidrógeno son las siguientes: reformado por vapor, oxidación parcial, reformado auto-térmico, electrólisis del agua, biomasa, proceso Kvaerner, proceso termoquímico, proceso fotoquímico, proceso fotobiológico. Los sistemas híbridos que producen energía eléctrica por medio de energía fotovoltaica tienen algunas desventajas debido a las condiciones variables del clima por un lado y a las dificultades relacionadas con el almacenamiento de energía eléctrica producida en exceso. El hidrógeno electrolítico podría ser la solución para nivelar el consumo esporádico y los picos de consumo, así como para el almacenamiento de energía eléctrica producida en exceso, seguido de la reintroducción de la energía en el sistema, cuando se requiera. Las soluciones tecnológicas convencionales más utilizadas para la producción de hidrógeno utilizando radiación solar son: un sistema de producción de hidrógeno mediante electrólisis del agua a temperatura ambiente y electricidad obtenida utilizando paneles fotovoltaicos cristalinos; un sistema de producción de hidrógeno mediante electrólisis del agua a temperatura ambiente y electricidad obtenida utilizando sistemas fotovoltaicos concentrados. Las soluciones tecnológicas de sistemas híbridos para la producción de hidrógeno utilizando radiación solar analizada en este artículo son: un sistema de producción de hidrógeno mediante electrólisis de vapor obtenida mediante una parte del espectro de la radiación solar y electricidad obtenida utilizando sistemas fotovoltaicos concentrados (sistema híbrido); un sistema de producción de hidrógeno mediante electrólisis de vapor obtenida utilizando sistemas solares térmicos concentrados y electricidad obtenida utilizando sistemas fotovoltaicos concentrados [1].

Existe un consenso a nivel mundial que las fuentes alternativas de energía deben ser desarrolladas. Esto surge a partir de la preocupación acerca de la capacidad de las fuentes de energía convencional para satisfacer las necesidades mundiales de energía y acerca de los efectos adversos en el ambiente que resultan de su utilización. Argumentos sólidos se han presentado a favor del hidrógeno como vector energético. En efecto, el hidrógeno podría ser utilizado como una fuente limpia y sostenible de energía, resolviendo entonces los problemas ambientales y de disponibilidad resultantes de las fuentes de energía convencionales. Además, el hidrógeno puede aliviar los problemas intrínsecos de los sistemas energéticos renovables, tales como la intermitencia, limitación asociada a la ubicación y desbalance entre la demanda y la oferta de energía. Como vector energético, el hidrógeno exhibe también una elevada densidad de masa energética y capacidad de almacenamiento a largo plazo y atributos de transporte a larga distancia de energía convencional. La energía del hidrógeno puede ser utilizada en diferentes campos, variando desde los sectores de transporte y de generación eléctrica hasta aplicaciones en viviendas. A nivel internacional, se proponen escenarios y programas importantes para el desarrollo e implementación de energía del hidrógeno. Sin embargo, un prerequisito para la penetración del mercado de hidrógeno como un vector energético es su disponibilidad a un precio competitivo. Sin embargo, el hidrógeno está disponible en la naturaleza principalmente combinado con otros elementos y debe por lo tanto ser producido. A la fecha, existen varios procesos de producción. Cada proceso requiere de una materia prima a partir del cual el hidrógeno es extraído y de una fuente de energía para permitir esta extracción. La materia prima podría ser cualquiera que contenga hidrógeno como el agua o un hidrocarburo. La energía podría ser convencional, renovable 0 de origen nuclear. Algunas de estas técnicas, tales como reformado de vapor y electrólisis de agua, han alcanzado etapa de madurez comercial; mientras que otras se encuentran aún en etapa experimental. La producción de hidrógeno utilizando técnicas basadas en energía solar y actualmente objeto de investigación intensa. En efecto, gracias a su disponibilidad y su carácter renovable y libre de contaminación, el hidrógeno solar es considerado como una de las alternativas que contribuyen a enfrentar el problema energético. Entre estas técnicas, la tecnología fotovoltaica de concentración merece atención especial [2].

Un sistema puede simultáneamente producir más de tres tipos de energía. Esto es posible debido a la 
concepción modular, denominada la concepción de módulos normalizados como una solución para atraer y concentrar la radiación solar, pero también diferente dependiendo del componente que convierte la energía. A inicio del año 2030 es posible que el precio de hidrógeno sea competitivo comparado con el precio del petróleo pesado. Los sistemas solares convencionales, ya sean fotovoltaicos o térmicos, generan solo un tipo de energía. Últimamente, existe una tendencia en el desarrollo de los sistemas de co-generación solar, denominada la producción simultánea de electricidad y calor. Desarrollos recientes en el campo solar revelan tendencias de desarrollo de sistemas de tri-generación que producen electricidad, calor y refrigeración. La producción de refrigeración utilizando calor a temperatura promedio $\left(90-95{ }^{\circ} \mathrm{C}\right)$ es posible utilizando enfriadores de absorción litio-bromato. Estas tecnologías son apropiadas para aplicaciones comerciales o residenciales. El desarrollo modular de sistemas solares híbridos concentrados permitirá el desarrollo de sistemas que generen varios tipos de energía. Ellos serán capaces de producir varios tipos de energía simultáneamente o dependiendo de las necesidades técnicas o ventajas económicas, en un proceso automatizado y programado [3].

Algunas de las fuentes primarias para la producción de hidrógeno son de tipo eléctrico, térmico, bioquímico, fotónico, electrotérmico, foto-eléctrico, y foto-bioquímico. Considerando los costos de producción y las eficiencias, el método photoelectroquímico y la electrólisis fotovoltaica resultan menos atractivos, por lo tanto, existiría se requiere para mejorar las eficiencias energéticas y exergéticas a fin de convertirlas en opciones potenciales frente a la producción de hidrógeno a partir de combustibles [4].

La potencia solar como instrumento para producir energía fue introducida hace 50 años y la tecnología se ha ido desarrollando drásticamente después de la aparición de los recursos fotovoltaicos. Aunque la energía solar es la fuente de energía más grande del planeta, menos del 0,06\% de la demanda global de electricidad es abastecida por energía solar. Es por eso que se está intentando aumentar el número de sistemas que combinan la energía solar con los sistemas de hidrógeno, en los que la energía fotovoltaica rompe las moléculas de agua en oxígeno e hidrógeno, que será usado después cuando el sol no esté incidiendo en la superficie terrestre. Es decir, el sistema fotovoltaico generará electricidad que será utilizada para la conversión de agua en moléculas de hidrógeno y oxígeno. Dicho hidrógeno será utilizado como vector energético, es decir, servirá de sistema de almacenamiento de energía. De esta manera, si la potencia obtenida a través a través de los paneles fotovoltaicos es mayor a la demandada en la red eléctrica, esa potencia sobrante se utilizará para el proceso de electrólisis de agua. Una vez obtenidos el hidrógeno y el oxígeno, el hidrógeno se almacenará para su futura utilización y el oxígeno se destinará a la atmósfera. En el momento en el que la demanda de la red sea superior a la obtenida a través de los paneles fotovoltaicos, el hidrógeno almacenado se utilizará para producir la potencia eléctrica necesaria. La transformación se realizará mediante la combustión del hidrógeno en motores de combustión interna alternativos conectados a generadores eléctricos o mediante el consumo de hidrógeno en pilas de combustible, entre otros procesos [5].

Las tecnologías de generación renovable son frecuentemente instaladas en edificios y distritos con el ánimo de disminuir sus emisiones de carbono y el consumo de energía no-renovable. Sin embargo, debido a un desbalance entre la oferta y demanda en escala horaria y estacional; una gran cantidad de energía es exportada a la red eléctrica en lugar de ser utilizada para reducir la demanda local. Una solución a esto es el almacenamiento local de electricidad para el auto-consumo posterior. Esto podría adicionalmente proporcionar a los distritos nuevas oportunidades de negocios, estabilidad financiera, flexibilidad y confiabilidad. En este artículo, la factibilidad de hidrógeno basado en almacenamiento eléctrico para un distrito es evaluada. El sistema energético del distrito incluye paneles fotovoltaicos y paneles híbridos. El sistema de almacenamiento consiste en la producción de hidrógeno utilizando la electricidad renovable generada dentro del distrito, almacenamiento de hidrógeno y uso posterior en una celda de combustible. La combinación de almacenamiento en batería conjuntamente con la conversión de energía y almacenamiento también es evaluada. Un enfoque de optimización multi-energético es utilizado para modelar el sistema. Los resultados del modelo son el tamaño óptimo de la batería, la capacidad del electrolizador, la capacidad de almacenamiento de hidrógeno, la capacidad de la celda de combustible y los flujos de energía a través del sistema. El modelo es también utilizado para comparar diferentes configuraciones de diseño del sistema [6].

Existen perspectivas críticas de innovación para la producción de hidrógeno que pueden ser 
introducidas bajo un concepto que incluye fuente, sistema, servicio, alcance, personal, réplica, seguridad, esquema, sector, solución, grupos de interés, normalización, subsidio, estimulación, estructura, estrategia, soporte y sostenibilidad. Los recursos energéticos renovables, en particular hidroenergía, geotermia y solar muestran un potencial único para respaldar sistemas innovadores de producción de hidrógeno [7].

Por lo tanto, se considera importante evaluar experimentalmente la producción de hidrógeno mediante electrólisis utilizando energía eléctrica producida por un sistema basado en energía solar, operando bajo condiciones locales, como parte de una línea de investigación acerca de la producción de hidrógeno a partir de energías renovables.

\section{Metodología}

La metodología a seguir consideró la evaluación preliminar de la irradiación solar disponible en la Facultad de Ingeniería Ambiental de la Universidad Nacional de Ingeniería (FIA-UNI). En ese sentido, se realizarán las mediciones correspondientes utilizando un radiómetro durante un período considerado como representativo en el contexto del presente proyecto. Según la Ref. [8], la irradiación solar, expresada en $\mathrm{kW} . \mathrm{h} / \mathrm{m}^{2}$-día, varía de acuerdo con el emplazamiento seleccionado y con el ángulo de inclinación sobre la superficie a utilizar, entre otros.

Seguidamente se procedió a la implementación de un sistema solar fotovoltaico, que consiste de un módulo fotovoltaico, una batería de acumulación, un controlador de carga y la instrumentación correspondiente para evaluar la energía eléctrica producida por el sistema solar fotovoltaico en función a la irradiación solar disponible.

En esta etapa se procedió a caracterizar la energía eléctrica producida por el sistema solar fotovoltaico bajo las condiciones de irradiación solar disponible en la FIA-UNI, identificando las variables principales que inciden en este proceso y la evaluación cuantitativa de los parámetros involucrados.

Seguidamente, se procedió a evaluar la cantidad de hidrógeno producida en función a la energía eléctrica utilizada y a la radiación solar disponible a fin de establecer el la eficiencia energética del proceso en forma integral De esta forma, el proceso experimental concluye, en la siguiente etapa, con la caracterización de las variables claves relacionadas con la producción de hidrógeno a partir de energía solar fotovoltaica, estableciendo los criterios correspondientes a la evaluación técnica de la eficiencia energética así como información preliminar para su evaluación económica.

Finalmente, se procedió a poner a punto un sistema demostrativo a fin de que pueda ser utilizado para fines educativos por los docentes y estudiantes de la FIA-UNI.

\section{Resultados}

En la Figura 1, se observan los valores máximos de temperatura cercanos a $30^{\circ} \mathrm{C}$ registrados en un día del mes de noviembre en la FIA-UNI.

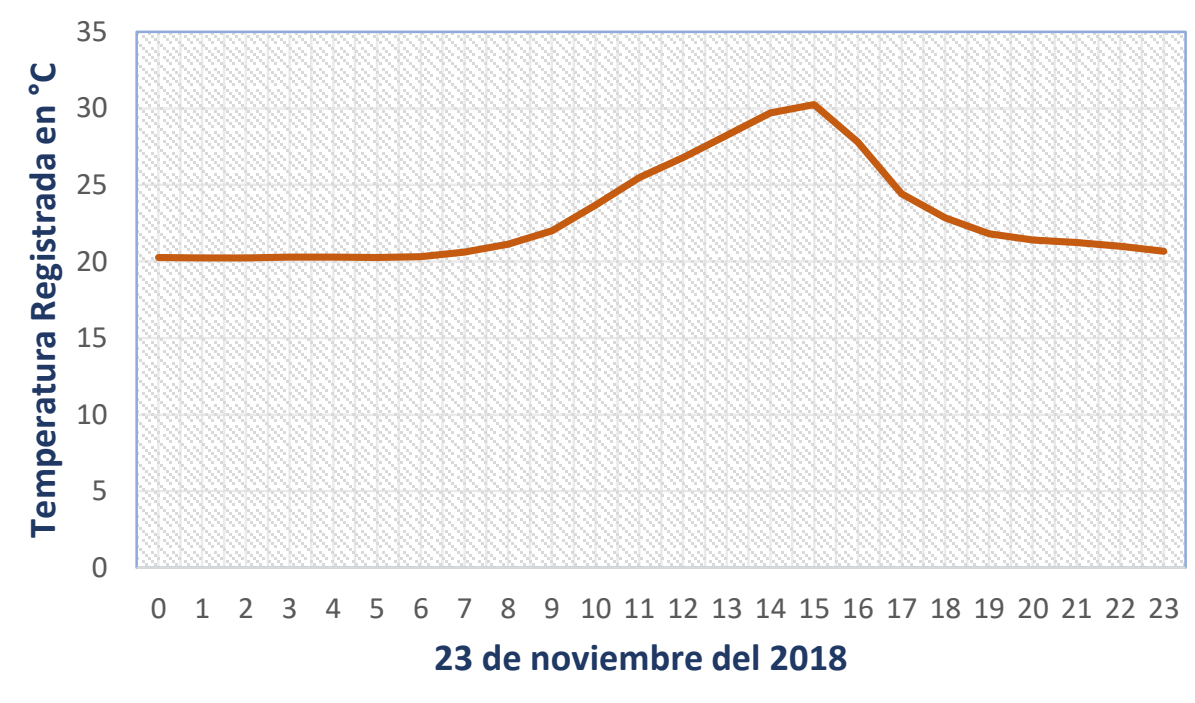

Figura 1: Temperatura Registrada en ${ }^{\circ} \mathrm{C}$ (FIA-UNI). 
En la Figura 2, se observan los valores mínimos de humedad relativa cercanos a $50 \%$ registrados en un día del mes de noviembre.
En Figura 3, se observan los valores máximos de irradiación solar cercanos a $900 \mathrm{~W} / \mathrm{m}^{2}$ registrados en un día del mes de marzo.

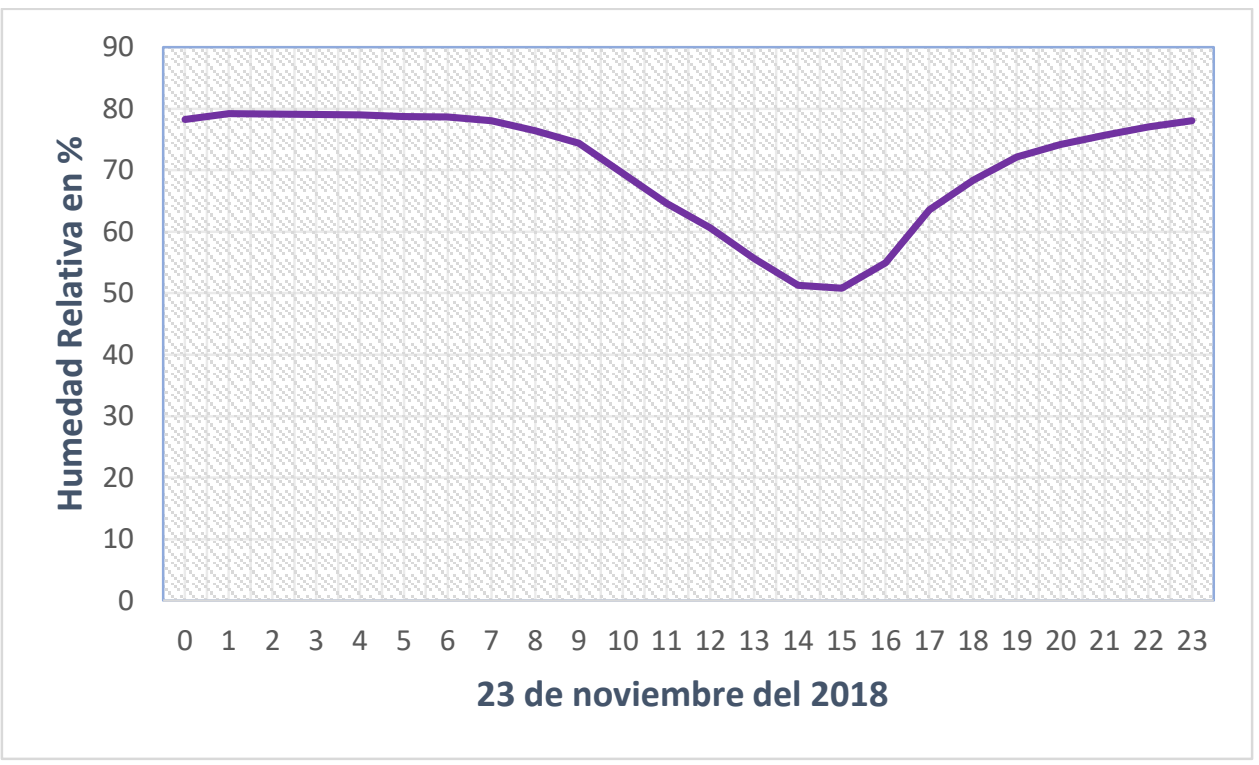

Figura 2: Humedad Relativa Registrada en \% (FIA-UNI).

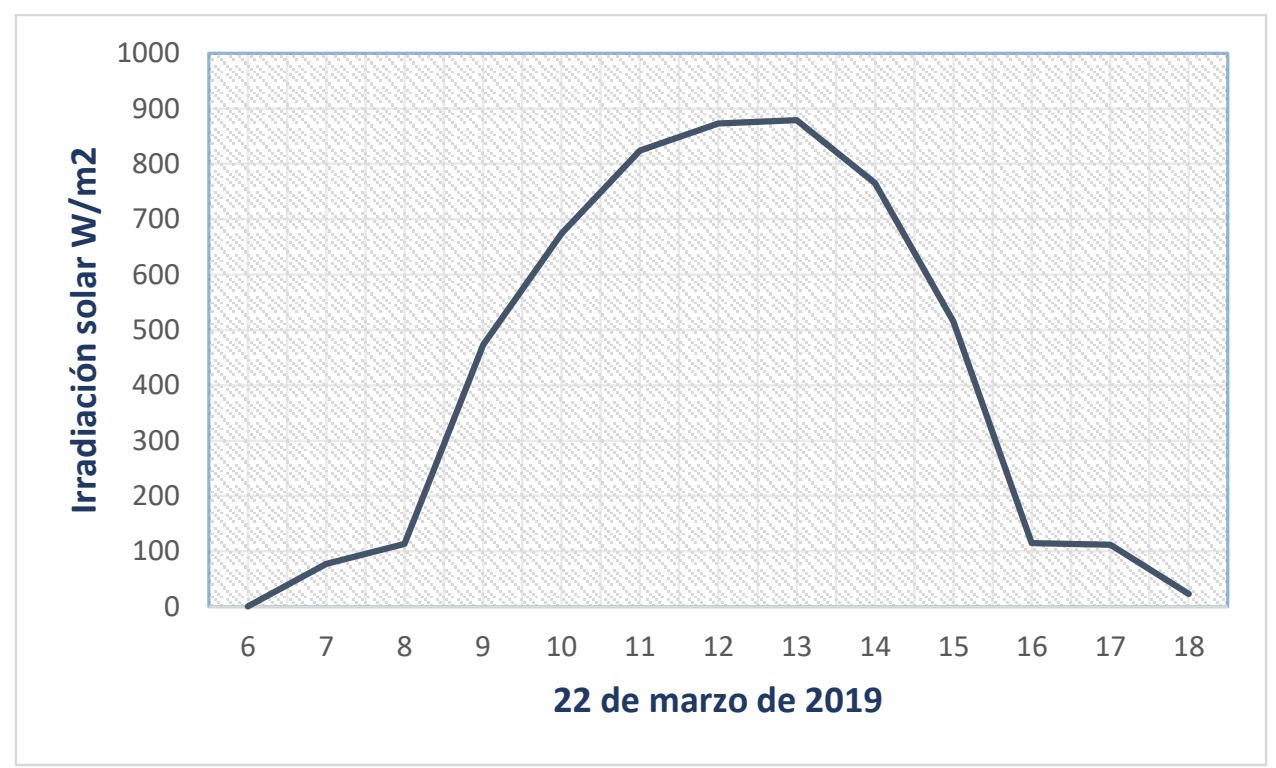

Figura 3: Irradiación Solar Registrada en W/m² (FIA-UNI).

Se implementó un sistema de producción de electricidad a partir del recurso solar. El sistema está compuesto de dos módulos fotovoltaicos, dos baterías, un inversor, y un controlador. El sistema se utilizará en adelante como módulo demostrativo como parte del proceso formativo de los alumnos de la FIA-UNI, según se muestra en la Figura 4.
Se hicieron pruebas de producción de hidrógeno, utilizando un sistema integrado provisto de una celda de combustible tipo PEM.

Se demostró que es posible obtener energía eléctrica a partir de los niveles de irradiación registrados en las instalaciones de la FIA-UNI. 
Se comprobó que, utilizando energía eléctrica, es posible producir hidrógeno mediante electrólisis, utilizando una celda de combustible.
En promedio, se obtuvo una ratio de $50,13 \mathrm{~kW} . \mathrm{h} / \mathrm{kg}$ de producción de hidrógeno a partir de energía solar fotovoltaica utilizando una celda de combustible tipo PEM.

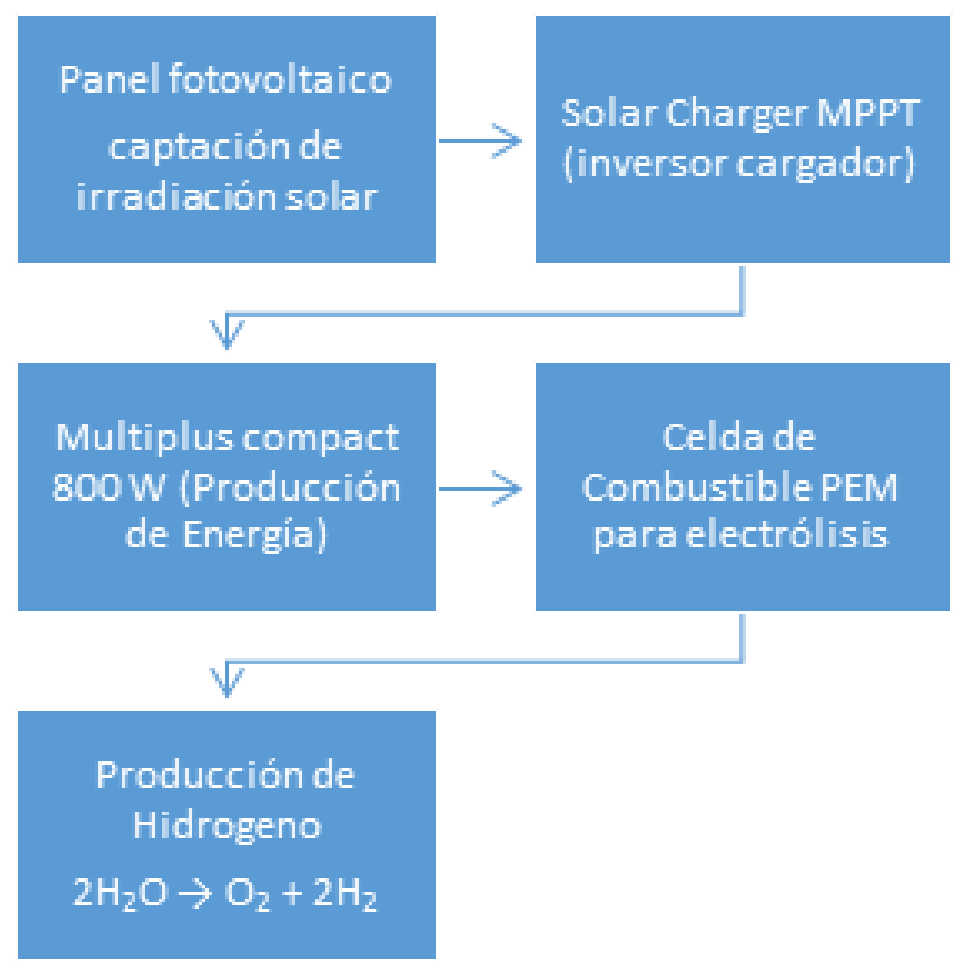

Figura 4: Esquema de Sistema Solar Fotovoltaico con Celda de Combustible implementado en la FIA-UNI.

\section{Conclusiones}

1. Se hicieron mediciones de temperatura en las instalaciones de la FIA-UNI. Se registraron valores máximos cercanos a los $30^{\circ} \mathrm{C}$ en un día del mes de noviembre.

2. Se hicieron mediciones de humedad relativa en las instalaciones de la FIA-UNI. Se registraron mínimos de $50 \%$ en un día del mes de noviembre.

3. Se hicieron mediciones de irradiación solar en las instalaciones de la FIA-UNI. Se registraron valores máximos cercanos a los $900 \mathrm{~W} / \mathrm{m}^{2}$, en un día del mes de marzo.

4. Se implementó un sistema de producción de electricidad a partir del recurso solar. El sistema está compuesto de dos módulos fotovoltaicos, dos baterías, un inversor, y un controlador. El sistema se utilizará como módulo demostrativo como parte del proceso formativo de los alumnos de la FIA-UNI.

5. Se hicieron pruebas de producción de hidrógeno, utilizando un sistema integrado provisto de una celda de combustible tipo PEM.
6. Se demostró que es posible obtener energía eléctrica a partir de los niveles de irradiación registrados en las instalaciones de la FIA-UNI.

7. Se comprobó que, utilizando energía eléctrica, es posible producir hidrógeno mediante electrólisis, utilizando una celda de combustible.

8. En promedio, se obtuvo una ratio de 50.13 kW.h/kg de producción de hidrógeno a partir de energía solar fotovoltaica utilizando una celda de combustible tipo PEM.

\section{Referencias}

[1] G. Badea et al., Energy Procedia 112 (2017) 418-425.

[2] R. Boudries, Energy Procedia 93 (2016) 96-101.

[3] G. Naghiu et al., Procedia Technology 22 (2016) $781-788$

[4] I. Dincer and C. Acar, International Journal of Hydrogen Energy 40 (2015) 1109411111. 
[5] Y. Gonzalez, MSc. Thesis, Universidad de Sevilla, 2016.

[6] A. Prasanna and V. Dorer, Energy Procedia 122 (2017) 373-378.

[7] I. Dincer and C. Acar, International Journal of Hydrogen Energy 42 (2017) 1484314864.

[8] Ministerio de Energía y Minas, Mapa de Energía Solar (Ministerio de Energía y Minas, Perú, 2003).

[9] J. Linares and B. Moratilla, El hidrógeno y la energía edited by Editorial Asociación Nacional de Ingenieros del ICAI (Madrid, España, 2007).
[10] I. Dincer and C. Zamfirescu, Sustainable Hydrogen Production edited by Editorial Elsevier (2016).

[11] International Energy Agency, Technology Roadmap. Hydrogen and Fuel Cells (Francia, 2015).

[12 M. Rose (Global Trends and Outlook for Hydrogen collaboration), IEA Hydrogen Technology Collaboration Program (2017).

E-mail: jnahuio@uni.edu.pe, ccamarenag@uni.pe, amen@uni.edu.pe 